\title{
Characteristic of free-rolling motion of two- dimensional rectangular body in the regular wave
}

\author{
H.J Kim / J. H. Jung / H.H. Chun \\ : Department of Naval Architecture and Ocean Engineering \\ Pusan National University \\ San 30, Jangjeon 2-Dong, Gumjeong-Gu \\ Busan 609-735, Korea \\ khyoju63@pusan.ac.kr / vof@pusan.ac.kr
}

\author{
H. S. Yoon \\ : Global Core Research Center for Ships and Offshore Plants \\ Pusan National University \\ San 30, Jangjeon 2-Dong, Gumjeong-Gu \\ Busan 609-735, Korea \\ lesmodel@pusan.ac.kr
}

\begin{abstract}
This study investigated the characteristics of rolling motion of rectangular body for regular waves with a range of wave periods that are equal to and longer than its natural roll period. Using the volume of fluid (VOF) method based on the finite volume method with standard $k-\varepsilon$ turbulence model, twodimensional (2D) incompressible viscous two-phase flow is simulated in a wave tank with the rectangular body. The present study introduces a wave period ratio $\left(T_{r}=T_{W} / T_{N}\right)$ of an incident wave period $\left(T_{W}\right)$ to the roll natural period $\left(T_{N}\right)$ of body. The wide range of $1 \leq \operatorname{Tr} \leq 2.5$ is considered in this study. In the wave periods considered in this study, the roll motion shows two distinct patterns. One is the single oscillatory motion which appears in the period ratio of $1 \leq \operatorname{Tr} \leq 1.7$. The other is the double oscillatory motion in $1.8 \leq \operatorname{Tr} \leq 2.5$. In these two regimes, flow and the roll motion of the rectangular body, which are induced by the fluid flow-structure interaction for various wave periods, are carefully investigated to reveal the mechanism of two roll modes.
\end{abstract}

\section{Keywords—regular wave; roll motion; wave}

\section{INTRODUCTION}

In the past several years offshore structures have moved to deeper water, growing interest in development of subsea resources. Therefore, offshore structures would undergo largeamplitude motion by harsh environments and extremely steep waves. Responses of floating bodies such as ships or offshore structures to incident waves are one of the main concerns in ocean engineering.

Offshore structures have been investigated in terms of six degree of freedom motion to evaluate design that can meet safety standards. As a result, there is an increasing interest in the use of numerical simulation to study the interaction between waves and structures. One of the most critical aspects of motions of a structure is roll, which is of practical interest for both safety and comfort reasons.

Roll motion, unlike other motions, is highly nonlinear because of the roll damping effect. However, the ability to predict roll motion lags considerably behind that of motions such as heave and pitch. Potential flow theories, based on the assumptions that the flow is inviscid and irrotational, can introduce large errors. They fail to account for viscous damping, flow separation, and vortex generation (Wehausen, 1971).

Most of the above publications are concerned with problems, either with fixed bodies or forced motion. Studies of the interaction between waves and free-rolling bodies are still very limited in the available literature.

Jung et al. [2] experimentally investigated wave interactions with a fixed rectangular structure using particle image velocimetry (PIV) to simulate the condition of a barge in a beam sea. The mean velocity field was demonstrated along with the generation and evolution of vortices on both sides of the structure. In subsequent studies, Jung [3] and Jung et al. [4] released the roll motion of the structure to investigate the twodimensional flow characteristics of wave interactions with freerolling rectangular structures using PIV.

In this study, we simulated the coupled interaction between a wave and a rectangular structure in rolling motion. The time variations of wave height and the roll motion of the rectangular structure were validated by comparison with experimental results of Jung et al. [2]. The main purpose of this present study is to investigate the effect of the longer wave period than the roll natural period of rectangular body. We focus on the characteristics of flow and the roll motion of the rectangular body which are induced by the fluid flow-structure interaction for various wave periods.

\section{NUMERICAL DETAILS}

\section{A. Numerical Approach}

The present two-dimensional problem of wave-structure interaction is governed by the Navier-Stokes equations and continuity equation. Once the Reynolds averaging approach for turbulence modeling is applied, the Navier-Stokes equations can be written in Cartesian tensor form as

$$
\frac{\partial}{\partial x_{i}}\left(\rho u_{i}\right)=0
$$




$$
\begin{aligned}
& \frac{\partial}{\partial t}\left(\rho u_{i}\right)+\frac{\partial}{\partial x_{j}}\left(\rho u_{i} u_{j}\right) \\
& =-\frac{\partial p}{\partial x_{i}}+\frac{\partial}{\partial x_{j}}\left[\mu\left(\frac{\partial u_{i}}{\partial x_{j}}+\frac{\partial u_{j}}{\partial x_{i}}\right)\right]+\frac{\partial}{\partial x_{j}}\left(-\rho \overline{u_{i}^{\prime} u_{j}^{\prime}}\right)+F_{i}
\end{aligned}
$$

where $x_{i}$ are Cartesian coordinates, $u_{i}$ are the corresponding velocity components, $p$ is the pressure, $\rho$ is the density, $\mu$ is the viscosity, and $F_{i}$ is external body force (e.g. gravity). Also, $-\rho \overline{u_{i}^{\prime} u_{j}^{\prime}}$ is the Reynolds stress term which has been closed by using the standard $k-\varepsilon$ turbulence model (Launder and Spalding [5]).

The equations of motion of rectangular body are expressed as follows,

$$
\begin{gathered}
\vec{F}=m \frac{d \vec{V}}{d t} \\
\vec{L}=\frac{d}{d t}\left(I_{0} \vec{\omega}\right)
\end{gathered}
$$

where $\vec{F}$ and $\vec{L}$ are hydrodynamic force and moment of hydrodynamic force acting on the body. Also, $\vec{V}$ and $\vec{\omega}$ are velocity and angular velocity of the floating body, respectively. $\mathrm{m}$ is the mass of the body, $I_{0}$ is the moment of inertia of the body at the center of gravity in space fixed coordinate system.

In this study, volume of fluid (VOF) method is employed to capture the free surface. VOF is popularly adopted to track and capture the free surface in two-phase problem. Also, most commercial CFD codes use a variation of the VOF approach.

In each cell, the volume fraction ( $Q_{\delta}$ ) of $\delta$ th fluid is introduced because this method is designed for two or more immiscible fluids. In each cell, the sum of volume fractions of all phases is unity ( $\sum_{\delta=1}^{n} Q_{\delta}=1$ ). A cell with a $Q_{\delta}$ value (scalar quantity) of 0 is void and a value of 1 represents a full cell. The cell contains a free surface if the $Q_{\delta}$ value is between 0 and 1 . At each time step, a transport equation is solved to find the distribution of the fluid. At each time step, the distribution of the fluid is found by solving following transport equation:

$$
\frac{\partial Q_{\delta}}{\partial t}+u_{i} \frac{\partial Q_{\delta}}{\partial x_{i}}=0
$$

A single momentum equation is solved throughout the domain, and the resulting velocity field is shared among the phases (Hirt and Nichols, [6]). Convection and diffusion terms are discretized using the QUICK (quadratic upstream interpolation for convective kinematics) scheme and the second order accurate central differencing scheme, respectively. For unsteady flow calculations, time derivative terms are discretized using the first order accurate backward implicit scheme. The velocity-pressure coupling and overall solution procedure are based on a PISO (pressure implicit with splitting of operators) algorithm adapted to structured grid. The commercial CFD package, FLUENT [7] is employed for all numerical predictions. Further details of the implementation can be found in FLUENT [7] manuals.

\section{B. Computational domain and Grid system}

A rectangular body that is $0.3 \mathrm{~m}$ wide (B), and $0.1 \mathrm{~m}$ high $(\mathrm{H})$ is used in the numerical simulation. The body is fixed at the center of gravity (CG) and is located at $20 \mathrm{~m}$ from the wave maker. The initial water height is $0.9 \mathrm{~m}$ and sloping beach is installed at the end of the wave tank to absorb the wave energy and reduce reflection as shown Fig. 1. A piston-type wave generator is incorporated in the computational domain to generate the desired incident waves. In order to move the piston, the layering mesh technique suitable for translation motion is employed. The piston motion is the pure surge (translation in the $\mathrm{x}$-direction only) which follows the sinusoidal function given as

$$
S(t)=A \cdot \sin (\omega t)
$$

where $A$ and $\omega$ are the amplitude and the frequency, respectively. The amplitude was changed as wave condition in this study.

To simulate the wave interaction with the body, the sliding mesh technique is used to handle the motion of the rectangular body induced by the fluid-structure interaction. So, two independent grid systems are generated based on the structured grid as shown in Fig. 2. The moving fluid zone including rectangular body is allowed to rotate by moment of hydrodynamic force acting on the body whereas the stationary fluid zone is fixed. These two flow fields are interpolated each other through the interface by using a linear interpolation method. The grid is refined near the body and free surface to achieve a better resolution of the flow field. Three different grid systems of the coarse, medium and fine grids having 60000, 100000 and 150000, respectively, are considered to test the grid dependence of the solutions and to validate the present numerical methods. The dependence of the solutions on the grid system in this study is not strong. Consequently, the medium grid system is selected for all cases.

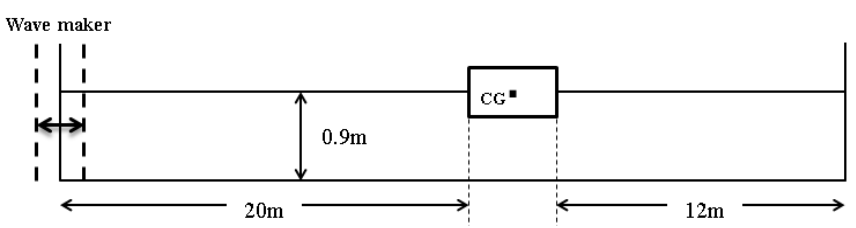

Fig. 1. Geometry of numerical wave tank and rectangular body 


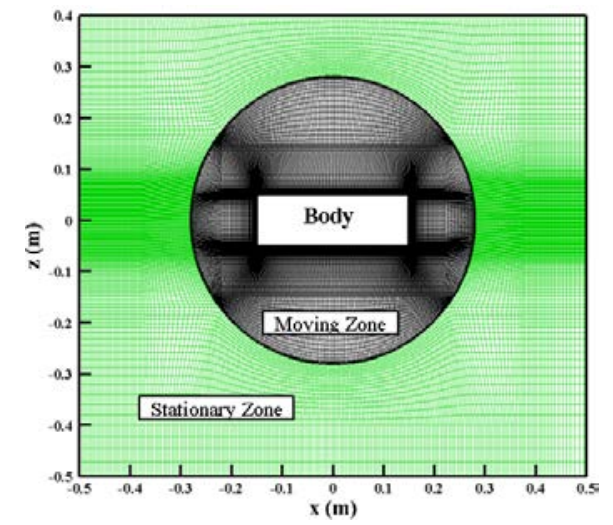

Fig. 2. Grid system

\section{Wave conditions}

The present study considers various wave periods that are equal to and longer than roll natural period of rectangular body to observe the dependence of the roll motion on the wave period. The roll natural period ( $T_{N}$ ) of body is $0.93 \mathrm{~s}$ and the corresponding roll natural frequency $\left(\omega_{N}\right)$ is $6.78 \mathrm{rad} / \mathrm{s}$, which are obtained by roll free decay test (Jung, [3]). The present study introduces a wave period ratio $\left(T_{r}=T_{W} / T_{N}\right)$ of an incident wave period ( $T_{W}$ ) to the roll natural period of body. The wide range of $1 \leq T_{r} \leq 2.5$ is considered in this study. Otherwise, the wave height is fixes as $0.04 \mathrm{~m}$.

\section{Validation}

Figure 3 shows a comparison of the profiles of wave elevation $(\eta)$ and the roll angle of the rectangular structure $(\phi)$ according to phases of velocity field with experimental results by Jung [3] for different wave periods of $T_{W}=0.93 \mathrm{~s}, T_{W}=1.2$ $\mathrm{s}$ and $T_{W}=2 \mathrm{~s}$. Regardless of the wave periods, the present results are in good agreement with those of the experiment with respect to the pattern, the value of the wave height, and the roll motion of the rectangular structure. The largest amplitude of $\phi$ appeared at $T_{W}=0.93 \mathrm{~s}$ due to the resonance effect, since this wave period was very close to the roll natural period of the rectangular structure.

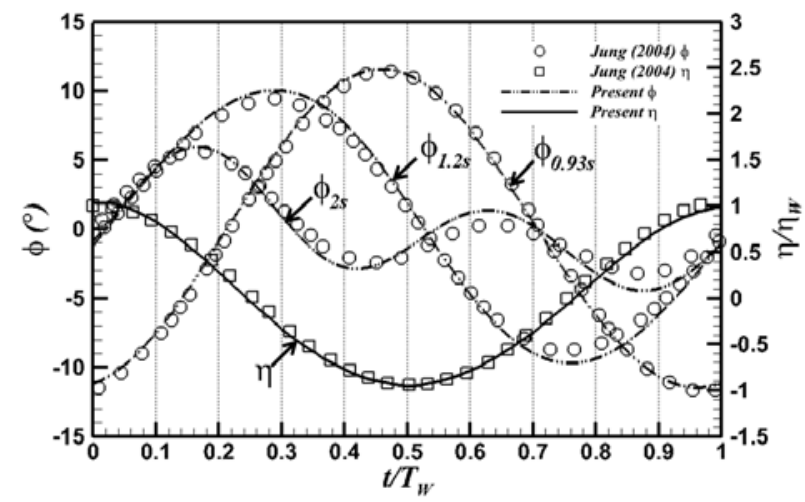

Fig. 3 Wave elevation ( $\eta$ ) and roll angle ( $\phi$ ) profiles during one period normalized by each wave period for $T_{W}=0.93 \mathrm{~s}, 1.2 \mathrm{~s}, 2 \mathrm{~s}$

\section{RESULTS AND DISCUSSION}

In the wave periods considered in this study, the roll motion shows two distinct patterns. One is the single oscillatory motion which appears in the period ratio of $1 \leq T_{r} \leq 1.7$. The other is the double oscillatory motion in $1.8 \leq T_{r} \leq 2.5$.

Thus, as the representative cases of the two patterns, $T_{r}=1$ and $T_{r}=2$ are considered in Fig. 4 which shows the profiles $(\eta)$ of incident waves without the body and the roll angle $(\phi)$ of the rectangular body during one period normalized by each wave period. For the roll angle, positive and negative values represent counter-clockwise and clockwise roll motions, respectively.

Fig. 5 shows vorticity contours according to the phase as marked in Fig. 4 for $T_{r}=1$ and $T_{r}=2$. First, for $T_{r}=1$, between phase (a) and (b) when the rotational angle of the body closest to the maximum value in the negative rotational direction, the positive vortices rotating counterclockwise are formed near the left bottom and the right side wall owing to the flow separation at the left and right bottom corners, respectively. At phase (b) where the body starts to rotate counterclockwise, the magnitude of the rotational angle decreases with the negative value and at between phases (c) and (d) the body is placed almost horizontally with about the rotational angle of $0^{\circ}$ as shown in Fig. 4. At phase (d), the body has almost the maximum value in the positive rotational angle with same amplitude to negative rotational direction. For $T_{r}=2$, at phase (a), the body has a counter-clockwise rotation and from the between phase (b) and (c) to when the body has a maximum rotational angle to phase (d), the body rotate clockwise. Then, the body starts to rotate counter-clockwise again with different amplitude relative to the first counterclockwise rotation. At phase (f), it continuously rotates clockwise with vorticies bigger strength than at phase (a). The wave period $T_{r}=1$, equal to roll natural period of the rectangular body, due to the resonance effect, has large amplitude of $\phi$ and strength of vortices in comparison with $T_{r}=2$.

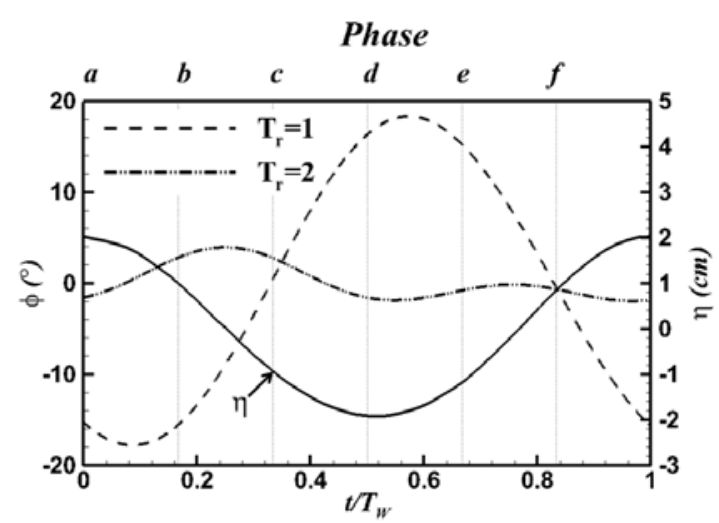

Fig. 4 Roll angle $(\phi)$ profiles with wave elevation $(\eta)$ during one period normalized by each wave period with phases of vorticity contour in Fig. 5 at the wave periods of $T_{r}=1$ and $T_{r}=2$ 

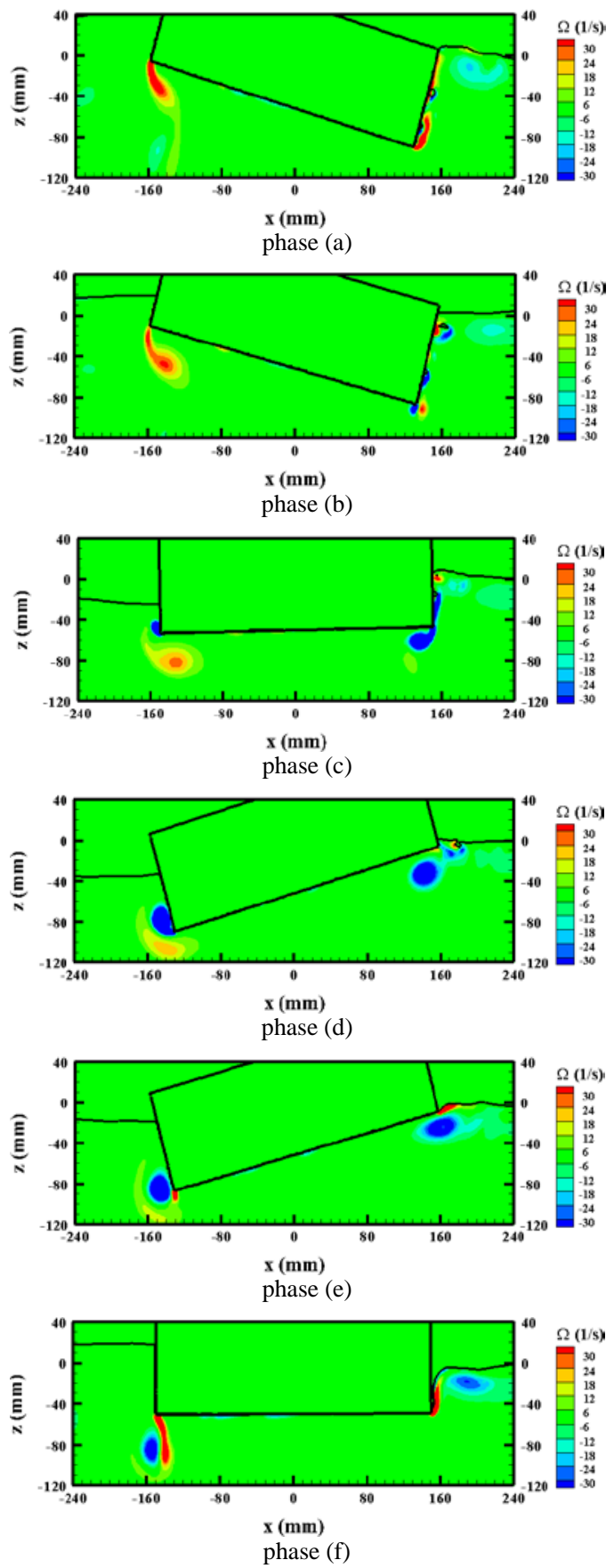

Fig. 5 Vorticity contours of $T_{r}=1$ (left column) and $T_{r}=2$ (right column): phase number of each subtitle matches to the phase in Fig. 4

In the region of period ratio $\left(1 \leq T_{r} \leq 1.7\right)$ where the body oscillate once during one wave period, the difference of maximum rotational angle between counter-clockwise and clockwise according to $T_{r}$. Fig. 6 shows time duration of counter-clockwise $\left(T_{+\phi}\right)$ and clockwise $\left(T_{-\phi}\right)$ roll motion during one period normalized by each wave period for $1 \leq T_{r} \leq 1.7$. According to time ratio, the time duration of counter-clockwise roll motion is longer than clockwise roll motion.
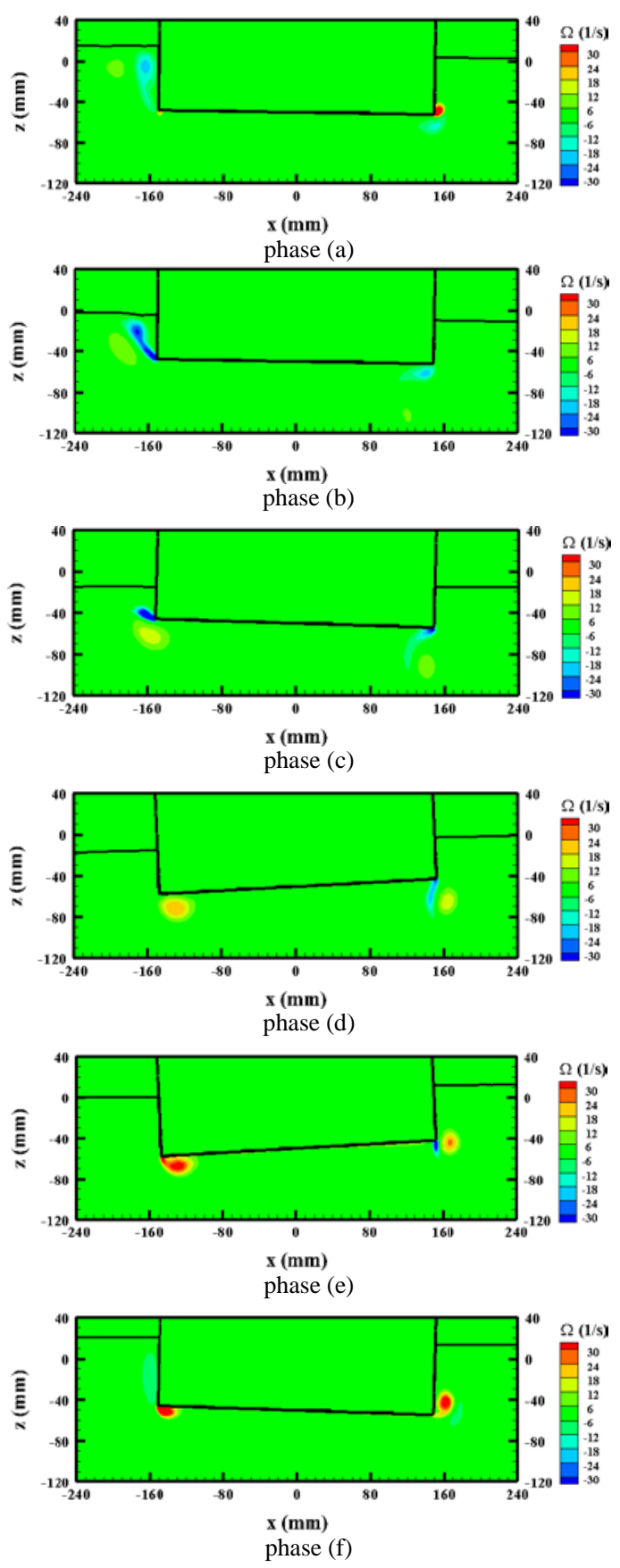

Fig. 5 Continued

Fig. 6 shows time duration of counter-clockwise $\left(T_{+\phi}\right)$ and clockwise $\left(T_{-\phi}\right)$ roll motions during one period normalized by each wave period for $1 \leq T_{r} \leq 1.7$ in which the body oscillates once during one wave period. The time duration difference between the counter-clockwise and clock roll motion becomes bigger with increasing $T_{r}$, which is supported by the roll angle $(\phi)$ of the rectangular body during one normalized period as early shown in Fig. 4. 


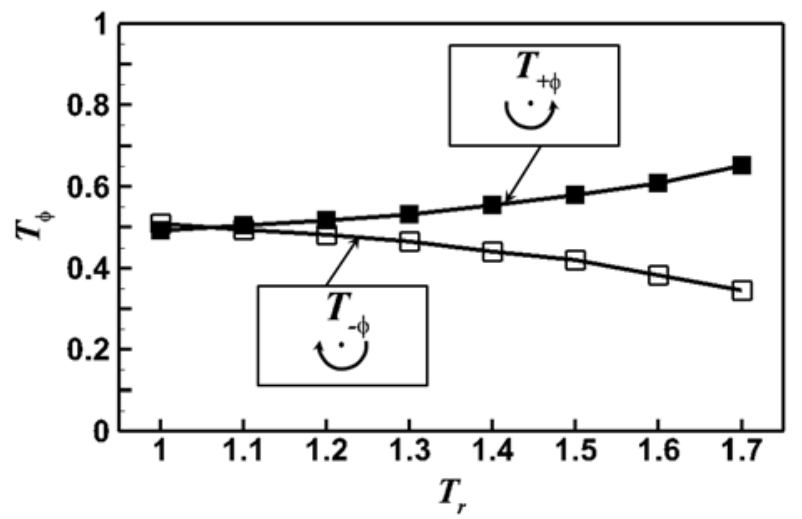

Fig. 6 Time duration of counter-clockwise $\left(T_{+\phi}\right)$ and clockwise $\left(T_{-\phi}\right)$ roll motion during one period normalized by each wave period for $1 \leq T_{r} \leq 1.7$

Fig. 7 shows the maximum roll angle ( $\left.\phi_{\text {Max }}\right)$ along the wave period ratio. The maximum roll angle decreases rapidly in the range of $1 \leq T_{r} \leq 1.5$. Further increasing $T_{r}$ from 1.5 to 2.5 , the decreasing rate is almost negligible since the wave slope is very smooth.

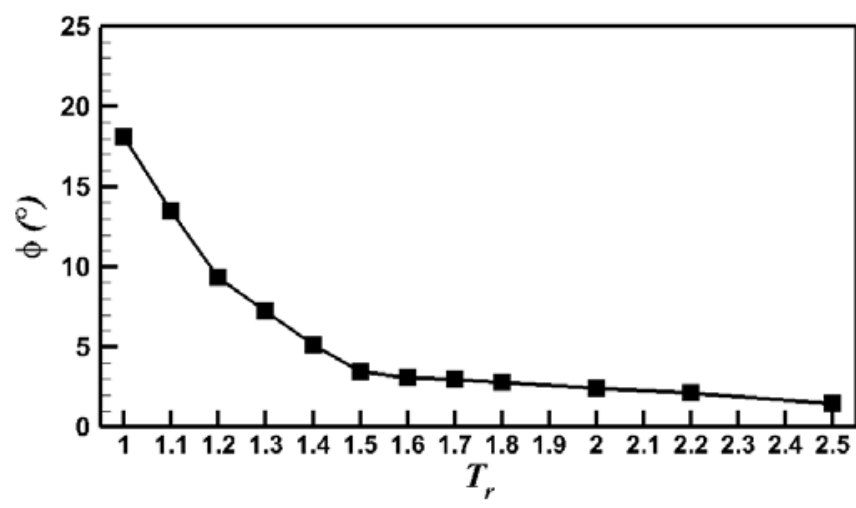

Fig. 7 Maximum roll angle according to $T_{r}$

\section{CONCLUSION}

This study investigated the characteristics of rolling motion of rectangular body for regular waves with a range of wave periods that are equal to and longer than its natural roll period. Using the volume of fluid (VOF) method based on the finite volume method with standard $k-\varepsilon$ turbulence model, twodimensional (2D) incompressible viscous two-phase flow is simulated in a wave tank with the rectangular body. The present study introduces a wave period ratio $\left(T_{r}=T_{W} / T_{N}\right)$ of an incident wave period $\left(T_{W}\right)$ to the roll natural period $\left(T_{N}\right)$ of body. The wide range of $1 \leq T_{r} \leq 2.5$ is considered in this study. In the wave periods considered in this study, the roll motion shows two distinct patterns. One is the single oscillatory motion which appears in the period ratio of $1 \leq T_{r} \leq 1.7$. The other is the double oscillatory motion in $1.8 \leq T_{r} \leq 2.5$.

\section{ACKNOWLEDGMENT}

This work was supported by the National Research Foundation of Korea (NRF) grant funded by the Korea government (MSIP) through GCRC-SOP (No. 2011-0030013) and (No. 2010-0025618)

\section{REFERENCES}

[1] J. V. Whhausen, "The motion of floating bodies," The Annual Review of Fluid Mechanics, vol. 3, pp. 237-268, 1971.

[2] K. H. Jung, K. A. Chang and E. T. Huang, "Two-dimensional flow characteristics of wave interactions with a fixed rectangular structure," Ocean Engineering, vol. 31, pp. 975-998, 2004a.

[3] K. H. Jung, Experimental study on rectangular barge in beam sea, Thesis for the degree of DOCTOR OF PHILOSOPHY, Texas A\&M University, 2004b.

[4] K. H. Jung, K. A. Chang, E. T. Huang, "Two-dimensional flow characteristics of wave interactions with a free-rolling rectangular structure," Ocean Engineering, vol. 32, pp. 1-20, 2005.

[5] B. E. Launder and D. B. Spalding, Lectures in Mathematical Models of Turbulence, Academic, London, England, 1972.

[6] C. W. Hirt and B. D. Nichols, "Volume of fluid (VOF) method for the dynamics of free boundaries," Journal of Computational Physics, vol. 39, pp. 201-225, 1981.

[7] FLUENT, FLUENT user's guide, ANSYS Inc, Lebanon, the USA, 2009.

\section{Creative Commons Attribution License 4.0 (Attribution 4.0 International, CC BY 4.0)}

This article is published under the terms of the Creative Commons Attribution License 4.0

https://creativecommons.org/licenses/by/4.0/deed.en US 